\title{
HAUSDORFF DIMENSION AND QUASICONFORMAL MAPPINGS
}

\author{
F. W. GEHRING $\dagger$ AND J. VÄISÄLÄ
}

Dedicated to the memory of A. S. Besicovitch

1. Introduction. In this paper we study what happens to the Hausdorff dimension of a set $A$, denoted by $\mathrm{H}$-dim $A$, under an $n$-dimensional quasiconformal mapping $f: D \rightarrow D^{\prime}$ with $A \subset D$. It is clear that

$$
\mathrm{H}-\operatorname{dim} f[A]=\mathrm{H}-\operatorname{dim} A
$$

if $f$ is a diffeomorphism or, more generally, if $f$ and $f^{-1}$ are locally Lipschitzian. We show first, however, that (1) need not hold if $f$ is a general quasiconformal mapping. Next we give bounds for $\mathrm{H}-\operatorname{dim} f[A]$ in terms of $\mathrm{H}-\operatorname{dim} A, n$, and the maximal dilatation of $f$. In particular, we prove that $\mathrm{H}-\operatorname{dim} A=0$ implies $\mathrm{H}-\operatorname{dim} f[A]=0$, and we conjecture that $\mathrm{H}-\operatorname{dim} A=n$ implies $\mathrm{H}-\operatorname{dim} f[A]=n$, or equivalently that H-dim $A<n$ implies $\mathrm{H}$-dim $f[A]<n$. We establish this conjecture for the case where $n=2$ and then prove that, for general $n, \mathrm{H}$-dim $f[A]<n$ whenever $A$ is contained in an $m$-dimensional hyperplane with $m<n$. An example shows that H-dim $f[A]$ can be arbitrarily close to $n$, even when $A$ is a 1-dimensional segment.

2. Notation. We shall use the terminology and notation for quasiconformal mappings given in [16]. Moreover, since we are concerned only with local properties which are invariant under Möbius transformations, we shall consider only quasiconformal mappings $f: D \rightarrow D^{\prime}$ where $D$ and $D^{\prime}$ are domains in the non-compact $n$-dimensional Euclidean space $R^{n}$.

For $a \in(0, \infty)$, the Hausdorff a-dimensional outer measure of a set $A \subset R^{n}$ is defined as

$$
H_{a}(A)=\lim _{d \rightarrow 0}\left(\inf \sum_{i} \operatorname{dia}\left(A_{i}\right)^{a}\right),
$$

where the infimum is taken over all countable coverings of $A$ by sets $A_{i}$ with $\operatorname{dia}\left(A_{i}\right)<d$. The Hausdorff dimension of $A$ is then given by

$$
\text { H-dim } A=\inf \left\{a: H_{a}(A)=0\right\} .
$$

Clearly $0 \leqslant \mathrm{H}$-dim $A \leqslant n$.

3. We shall need the following generalization of a result due to Mori [13; Lemma 4].

Lemma. Suppose that $f: D \rightarrow D^{\prime}$ is an $n$-dimensional $K$-quasiconformal mapping, that $U$ is a bounded domain with $\bar{U} \subset D$, and that $x \in U$. Let

$$
M=\max _{y \in \partial U}|y-x|, m=\min _{y \in \partial U}|y-x|, L=\max _{y \in \partial U}|f(y)-f(x)|, l=\min _{y \in \partial U}|f(y)-f(x)| .
$$

Received 4 November, 1971.

$\uparrow$ This research was supported in part by the National Science Foundation, Contracts GP 7041X and GP 28115. 
If the ball $B^{n}(f(x), L)$ is contained in $D^{\prime}$, then

$$
L \leqslant C l \text {, }
$$

where $C$ is a finite constant which depends only on $n, K$, and $M / m$.

Proof. Suppose that $l<L$ and let $R$ denote the image under $f^{-1}$ of the spherical ring

$$
R^{\prime}=\{y: l<|y-f(x)|<L\} \subset D^{\prime} .
$$

Then $R$ is a ring which separates $x$ and a point $y \in \partial U$ from $\infty$ and a point $z \in \partial U$. Hence if $\Gamma$ is the family of arcs joining the components of $C(R)$ in $R$, it follows from the extremal property of the Teichmüller ring in $R^{n}[4$ and 14$]$, or from $[16 ; 11.9]$ that

$$
M(\Gamma) \geqslant h_{n}\left(\frac{|z-x|}{|y-x|}\right) \geqslant h_{n}\left(\frac{M}{m}\right),
$$

where $h_{n}:(0, \infty) \rightarrow(0, \infty)$ is positive and decreasing. Then since $f$ is $K$-quasiconformal

$$
M(\Gamma) \leqslant K M(f[\Gamma])=K \omega_{n-1}\left(\log \frac{L}{l}\right)^{1-n},
$$

and (4) follows from (5) and (6).

4. The Cantor sets $C_{s}{ }^{n}$. For each integer $n \geqslant 1$ and each $s \in\left(0, \frac{1}{2}\right)$ we define a family of Cantor sets $C_{s}{ }^{n}$ as follows. Let $Q$ denote the closed unit cube

$$
Q=\left\{x=\left(x_{1}, \ldots, x_{n}\right): 0 \leqslant x_{i} \leqslant 1\right\}
$$

choose a collection of $2^{n}$ disjoint closed cubes $Q_{i}$ of side $s$ in int $Q, 1 \leqslant i \leqslant 2^{n}$, oriented so that for each $i$ there exists a similarity mapping

$$
g_{i}(x)=s x+a_{i}, a_{i} \in Q,
$$

which maps $Q$ onto $Q_{i}$. Such collections of cubes $Q_{i}$ obviously exist for each $s \in\left(0, \frac{1}{2}\right)$. Next for each $j \geqslant 1$ let

$$
F_{j}=\bigcup_{i_{1}, \ldots, i_{j}=1}^{2^{n}} g_{i_{1}} \circ \ldots \circ g_{i j}[Q]
$$

Then $\left\{F_{j}\right\}$ is a decreasing sequence of compact sets, and each set $F_{j}$ is the union of $2^{j n}$ disjoint closed cubes of side $s^{j}$. Hence

$$
C_{s}^{n}=\bigcap_{j=1}^{\infty} F_{j}
$$

is a compact set, and

$$
\mathrm{H}-\operatorname{dim} C_{s}{ }^{n}=n \frac{\log \frac{1}{2}}{\log s}
$$

by, for example, [1; Theorem 3] or [12; Theorem III]. In particular,

$$
0<\mathrm{H}-\operatorname{dim} C_{s}^{n}<n
$$


and

$$
\lim _{s \rightarrow 0} \mathrm{H}-\operatorname{dim} C_{s}^{n}=0, \lim _{s \rightarrow \frac{1}{2}} \mathrm{H}-\operatorname{dim} C_{s}^{n}=n .
$$

5. THEOREM. For each integer $n \geqslant 2$ and each pair of such Cantor sets $C_{s}{ }^{n}$ and $C_{t}^{n}$ there exists a quasiconformal mapping $f: R^{n} \rightarrow R^{n}$ which maps $C_{s}{ }^{n}$ onto $C_{t}{ }^{n}$.

Proof. Let $g_{i}$ and $F_{j}, g_{i}{ }^{\prime}$ and $F_{j}{ }^{\prime}$ denote respectively the similarity mappings and sets corresponding to the constructions for $C_{s}{ }^{n}, C_{t}{ }^{n}$ given in $\$ 4$. Then it is not difficult to see that there exists a piecewise linear homeomorphism $f_{1}: R^{n} \rightarrow R^{n}$ such that $f_{1}(x)=x$ if $x \in R^{n} \sim Q$ and such that for each $i$

$$
f_{1}(x)=g_{i}{ }^{\prime} \circ g_{i}^{-1}(x)
$$

if $x \in g_{i}[Q]$. Then $f_{1}$ is $K$-quasiconformal for some $K$ and $f_{1}\left[F_{1}\right]=F_{1}{ }^{\prime}$. Next define $f_{2}: R^{n} \rightarrow R^{n}$ by setting $f_{2}(x)=f_{1}(x)$ if $x \in R^{n} \sim F_{1}$ and

$$
f_{2}(x)=g_{i}{ }^{\prime} \circ f_{1} \circ g_{i}^{-1}(x)
$$

if $x \in g_{i}[Q]$. Then $f_{2}$ is a piecewise linear $K$-quasiconformal mapping, $f_{2}\left[F_{2}\right]=F_{2}{ }^{\prime}$, and for each $i$ and $j$

$$
f_{2}(x)=g_{i}^{\prime} \circ g_{j}^{\prime} \circ g_{j}^{-1} \circ g_{i}^{-1}(x)
$$

if $x \in g_{i} \circ g_{j}[Q]$. Continuing in this way, we obtain a sequence of piecewise linear $K$-quasiconformal mappings $f_{j}: R^{n} \rightarrow R^{n}$ such that $f_{j+1}(x)=f_{j}(x)$ in $R^{n} \sim F_{j}$ and $f_{j}\left[F_{j}\right]=F_{j}{ }^{\prime}$. This sequence converges to a $K$-quasiconformal mapping $f: R^{n} \rightarrow R^{n}$ which maps $F_{j}$ onto $F_{j}{ }^{\prime}$ for each $j$. Hence $f$ maps $C_{s}{ }^{n}$ onto $C_{t}{ }^{n}$.

6. CoRollary. For each integer $n \geqslant 2$ and each pair of numbers $\alpha, \beta \in(0, n)$, there exists a quasiconformal mapping $f: R^{n} \rightarrow R^{n}$ and a compact set $A \subset R^{n}$ such that

$$
\mathrm{H}-\operatorname{dim} A=\alpha, \mathrm{H}-\operatorname{dim} f[A]=\beta .
$$

Proof. By (7) and (8) we can choose $s, t \in\left(0, \frac{1}{2}\right)$ so that for any of the corresponding Cantor sets $C_{s}{ }^{n}, C_{t}{ }^{n}$,

$$
\mathrm{H}-\operatorname{dim} C_{s}{ }^{n}=\alpha, \mathrm{H}-\operatorname{dim} C_{t}{ }^{n}=\beta \text {. }
$$

Theorem 5 then yields a quasiconformal mapping $f: R^{n} \rightarrow R^{n}$ which maps $C_{s}{ }^{n}$ onto $C_{t}{ }^{n}$, and (9) follows with $A=C_{s}{ }^{n}$.

7. Remark. The above proof shows that for each $\alpha \in(0, n)$ there exists a set $A \subset R^{n}$ with $\mathrm{H}-\operatorname{dim} A=\alpha$ such that

$$
\inf _{f} \mathrm{H}-\operatorname{dim} f[A]=0, \sup _{f} \mathrm{H}-\operatorname{dim} f[A]=n,
$$

where the infimum and supremum are taken over all quasiconformal mappings $f: D \rightarrow D^{\prime}$ with $A \subset D$. We consider next what can be said if we take the infimum and supremum in (10) over the subclass of mappings $f: D \rightarrow D^{\prime}$ which are $K$ quasiconformal for some fixed $K$.

8. THEOREM. If $f: D \rightarrow D^{\prime}$ is an $n$-dimensional $K$-quasiconformal mapping and if $A \subset D$ with $\mathrm{H}-\operatorname{dim} A \geqslant \alpha>0$, then $\mathrm{H}-\operatorname{dim} f[A] \geqslant \beta>0$, where

$$
\beta=\alpha K^{1 /(1-n)} \geqslant \alpha / K \text {. }
$$

Proof. Since $A$ is the countable union of sets with compact closure in $D$, we may assume that $A$ is contained in a compact subset of $D$. Then since $f^{-1}$ is locally 
Hölder continuous with exponent $K^{1 /(1-n)}$ in $D^{\prime}([5$; Corollary 6$]$ or $[10 ; 3.2])$, there exists a positive constant $c$ such that

$$
|f(x)-f(y)| \geqslant c|x-y|^{K^{1 /(n-1)}}
$$

for all $x, y \in A$. If $b>\mathrm{H}-\operatorname{dim} f[A]$, then (2), (3), and (12) imply that $H_{a}(A)=0$, where $a=b K^{1 /(n-1)}$. Hence $a \geqslant \alpha$ and (11) follows.

9. CoRollary. If $f: D \rightarrow D^{\prime}$ is an n-dimensional quasiconformal mapping and if $A \subset D$ with $\mathrm{H}-\operatorname{dim} A=0$, then $\mathrm{H}-\operatorname{dim} f[A]=0$.

10. CONJECTURE. If $f: D \rightarrow D^{\prime}$ is an $n$-dimensional $K$-quasiconformal mapping and if $A \subset D$ with $\mathrm{H}-\operatorname{dim} A \leqslant \alpha<n$, then $\mathrm{H}-\operatorname{dim} f[A] \leqslant \beta<n$, where $\beta$ depends only' on $\alpha, n$, and $K$.

11. We shall establish this conjecture for the case where $n=2$. The proof is based on the following important result due to Bojarski [7; p. 226].

THEOREM. If $f$ is a 2-dimensional $K$-quasiconformal mapping, then its Jacobian $J_{f}$ is locally $L^{q}$-integrable for $q \in[1, p(K)]$, where $p(K)>1$ depends only on $K$.

It is easy to see that $p(K) \leqslant K /(K-1)$, and it has been conjectured that Theorem 11 holds with $p(K)=K /(K-1)$.

12. THEOREM. If $f: D \rightarrow D^{\prime}$ is a 2-dimensional $K$-quasiconformal mapping and if $A \subset D$ with $\mathrm{H}-\operatorname{dim} A \leqslant \alpha<2$, then $\mathrm{H}-\operatorname{dim} f[A] \leqslant \beta<2$, where

$$
\beta=\frac{2 p(K) \alpha}{2(p(K)-1)+\alpha}
$$

and $p(K)$ is the constant given in Theorem 11.

Proof. As in the proof of Theorem 8, we may assume that $A$ is contained in an open set with compact closure $F$ in $D$. Then for each $a \in(\alpha, 2)$ and each $q \in(1, p(K))$ we must show that $H_{b}(f[A])=0$, where

$$
b=\frac{2 q a}{2(q-1)+a} .
$$

Choose $\varepsilon>0$ and $d>0$. Then $H_{a}(A)=0$ and by [8; Lemma 1] we can choose a covering of $A$ by non-overlapping squares $Q_{i}$ of side $s_{i}$ such that $Q_{i} \subset F$,

$$
\operatorname{dia}\left(f\left[Q_{i}\right]\right)<d,
$$

and

$$
\sum_{i} s_{i}{ }^{a}<\varepsilon
$$

Let $x_{i}$ denote the centre of $Q_{i}$ and set

$$
L_{i}=\max _{y \in \partial Q_{i}}\left|f(y)-f\left(x_{i}\right)\right|, l_{i}=\min _{y \in \partial Q_{i}}\left|f(y)-f\left(x_{i}\right)\right| .
$$

By choosing $d$ sufficiently small, we may assume that the disks $B^{2}\left(f\left(x_{i}\right), L_{i}\right)$ all lie in $D^{\prime}$. Then Lemma 3 implies that $L_{i} \leqslant C l_{i}$, where $C$ is a finite constant which depends only on $K$. Hence

$$
\operatorname{dia}\left(f\left[Q_{i}\right]\right) \leqslant 2 L_{i} \leqslant 2 C l_{i} \leqslant C_{1} m\left(f\left[Q_{i}\right]\right)^{1 / 2},
$$


where $C_{1}=2 C \pi^{-1 / 2}$. On the other hand,

$$
m\left(f\left[Q_{i}\right]\right)=\int_{Q_{i}} J_{f} d m \leqslant s_{i}^{2(q-1) / q}\left(\int_{Q_{i}} J_{f}^{q} d m\right)^{1 / q}
$$

by Hölder's inequality. Thus

$$
\sum_{i} \operatorname{dia}\left(f\left[Q_{i}\right]\right)^{b} \leqslant C_{1}{ }^{b} \sum_{i} s_{i}^{b(q-1) / q}\left(\int_{Q_{i}} J_{f}^{q} d m\right)^{b /(2 q)},
$$

and a second application of Hölder's inequality yields

$$
\sum_{i} \operatorname{dia}\left(f\left[Q_{i}\right]\right)^{b} \leqslant C_{1}{ }^{b}\left(\sum_{i} s_{i}^{a}\right)^{b(q-1) /(a q)}\left(\int_{F} J_{f}^{q} d m\right)^{b /(2 q)} .
$$

Finally, since $d$ can be chosen arbitrarily small, (14) and (15) imply that

$$
H_{b}(f[A]) \leqslant C_{1}{ }^{b}\left(\int_{F} J_{f}^{q} d m\right)^{b /(2 q)} \varepsilon^{b(q-1) /(a q)},
$$

and letting $\varepsilon \rightarrow 0$ yields $H_{b}(f[A])=0$.

13. COROLLARY. If $f: D \rightarrow D^{\prime}$ is a 2-dimensional quasiconformal mapping and if $A \subset D$ with $\mathrm{H}-\operatorname{dim} A=2$, then $\mathrm{H}-\operatorname{dim} f[A]=2$.

14. Remark. If the conjecture that Theorem 11 holds with $p(K)=K /(K-1)$ is correct, then Theorem 12 would imply that

$$
\frac{2 \alpha}{2 K-(K-1) \alpha} \leqslant \mathrm{H}-\operatorname{dim} f[A] \leqslant \frac{2 K \alpha}{2+(K-1) \alpha}
$$

for each 2-dimensional $K$-quasiconformal mapping $f: D \rightarrow D^{\prime}$ and each set $A \subset D$ with $\mathrm{H}-\operatorname{dim} A=\alpha$. These bounds are asymptotic to those implied by Theorem 8 as $\alpha \rightarrow 0$.

15. Suppose that $f: D \rightarrow D^{\prime}$ is an $n$-dimensional quasiconformal mapping and that $J_{f}$ is locally $L^{q}$-integrable for $q \in[1, p)$ where $p>1$. Then the proof for Theorem 12 shows that

$$
\mathrm{H}-\operatorname{dim} f[A] \leqslant \beta=\frac{n p \alpha}{n(p-1)+\alpha}<n
$$

for each $A \subset D$ with H-dim $A \leqslant \alpha<n$. Unfortunately it is not known whether the analogue of Theorem 11 holds in higher dimensions, and hence we cannot use this argument to establish Conjecture 10 for general $n$.

We can, however, establish a weaker form of Conjecture 10 for general $n$ by a different method. We require some additional notation. Suppose that $f: D \rightarrow D^{\prime}$ is an $n$-dimensional homeomorphism. If $\bar{B}^{n}(x, r) \subset D$, we set

$$
L(x, f, r)=\max _{|y-x|=r}|f(y)-f(x)|, l(x, f, r)=\min _{|y-x|=r}|f(y)-f(x)| .
$$

Next we say that a closed cube $Q \subset D^{\prime}$ is $f$-admissible if for each $x \in f^{-1}[Q]$,

$$
\bar{B}^{n}(x, d) \subset D, \bar{B}^{n}(f(x), L(x, f, d)) \subset D^{\prime}
$$


where $d=\operatorname{dia}\left(f^{-1}[Q]\right)$. Since $f$ is a homeomorphism, each point of $D^{\prime}$ is contained in the interior of some $f$-admissible cube $Q$.

16. Lemma. Suppose that $f: D \rightarrow D^{\prime}$ is an $n$-dimensional $K$-quasiconformal mapping, that $T$ is an (n-1)-plane in $R^{n}$, and that $Q$ is an $f$-admissible closed cube of side $s$ in $D^{\prime}$. Then there exists an integer $p \geqslant 2$, which depends only on $n$ and $K$, such that the subdivision of $Q$ into $p^{n}$ congruent closed cubes of side $s / p$ contains a cube which does not meet $f[D \cap T]$.

Proof. Let $C=C(n, K)$ denote the number given by Lemma 3 when $M / m=1$. We shall show that the assertion is true for $p>\max \left(6,3 C n^{1 / 2}\right)$.

Fix such an integer $p$, let $Q_{0}$ denote a cube of the corresponding subdivision which contains the centre of $Q$, and let $S=f[D \cap T]$. If $S \cap Q_{0}=\varnothing$, we are finished. Otherwise choose a point $z \in S \cap Q_{0}$, let $y=f^{-1}(z)$, and let $e$ denote a unit normal to $T$. Then $B=B^{n}(z, s / 3) \subset Q$ and we can choose $r>0$ so that

$$
x=y+r e \in f^{-1}[\partial B] \subset f^{-1}[Q] .
$$

Since $Q$ is $f$-admissible, Lemma 3 implies that

$$
l(x, f, r) \geqslant(1 / C) L(x, f, r) \geqslant(1 / C)|f(y)-f(x)|=s /(3 C) .
$$

Next since $T$ is an (n-1)-plane, $B^{n}(x, r) \cap T=\varnothing$ while

$$
B^{n}(f(x), s /(3 C)) \subset f\left[B^{n}(x, r)\right] .
$$

Hence the ball $B^{n}(f(x), s /(3 C))$ does not meet $S$, and since this ball contains a cube of the subdivision, the proof is complete.

17. Definition. A set $S \subset R^{n}$ is said to be a $K$-quasiconformal $m$-ball if there is a neighbourhood $D$ of $S$ and an $n$-dimensional $K$-quasiconformal mapping $f: D \rightarrow D^{\prime}$ such that $f[S]$ is an ordinary $m$-dimensional (open or closed) ball. When $m=1$, $S$ is also said to be a $K$-quasiconformal arc. Finally $S$ is said to be a quasiconformal $m$-ball if it is a $K$-quasiconformal $m$-ball for some $K$.

18. THEOREM. If $S$ is a $K$-quasiconformal $m$-ball in $R^{n}$ and if $m<n$, then

$$
m \leqslant \mathrm{H}-\operatorname{dim} S \leqslant \beta<n,
$$

where $\beta$ depends only on $n$ and $K$.

Proof. Since $S$ is homeomorphic to an ordinary $m$-ball, $S$ has topological dimension $m$, and the lower bound in (16) follows from [6; p. 107].

For the upper bound, there exists, by hypothesis, an $n$-dimensional $K$-quasiconformal mapping $f: D \rightarrow D^{\prime}$ such that $S \subset f[D \cap T]$ for some $(n-1)$-plane $T \subset R^{n}$. Choose $p=p(n, K)$ as in Lemma 16 and set

Then

$$
a=\left(1-p^{-n}\right)^{1 / 2}<1 \text {. }
$$

$$
a p^{n}>a^{2} p^{n}=p^{n}-1,
$$

and we may choose $\beta \in(0, n)$ so that $a p^{\beta}=p^{n}-1$. Then $\beta$ depends only on $n$ and $K$, and it suffices to show that $H_{\beta}(S)=0$. Moreover since $S$ can be covered by a countable collection of $f$-admissible cubes, it suffices to prove that $H_{\beta}(S \cap Q)=0$ for each $f$-admissible closed cube $Q \subset D^{\prime}$.

Let $Q$ denote such a cube with side $s$, subdivide $Q$ into $p^{n}$ congruent closed cubes 
of side $s / p$, and let $Q_{1}, \ldots, Q_{q}$ denote the cubes of this subdivision which meet $S$. Since $Q$ is $f$-admissible, Lemma 16 implies that $q \leqslant p^{n}-1$ and hence that

$$
\sum_{i=1}^{q} \operatorname{dia}\left(Q_{i}\right)^{\beta}=q\left((s / p) n^{1 / 2}\right)^{\beta} \leqslant a\left(s n^{1 / 2}\right)^{\beta} .
$$

Next subdivide each cube $Q_{i}$ into $p^{n}$ congruent closed cubes of side $s / p^{2}$, and let $Q_{i 1}, \ldots, Q_{i q_{t}}$ denote the cubes of this subdivision which meet $S$. Then since each $Q_{i}$ is $f$-admissible, Lemma 16 implies that $q_{i} \leqslant p^{n}-1$ for each $i$ and hence that

$$
\sum_{i=1}^{q}\left(\sum_{j=1}^{q_{l}} \operatorname{dia}\left(Q_{i j}\right)^{\beta}\right)=\sum_{i=1}^{q} q_{i}\left(\left(s / p^{2}\right) n^{1 / 2}\right)^{\beta} \leqslant a^{2}\left(s n^{1 / 2}\right)^{\beta} .
$$

Continuing in this way, we see that for each integer $j \geqslant 1, S \cap Q$ can be covered by a finite number of closed cubes $Q_{i}{ }^{\prime}$ of side $s / p^{j}$ such that

$$
\sum_{i} \operatorname{dia}\left(Q_{i}{ }^{\prime}\right)^{\beta} \leqslant a^{j}\left(s n^{1 / 2}\right)^{\beta} .
$$

Then letting $j \rightarrow \infty$ we conclude that $H_{\beta}(S \cap Q)=0$.

19. COROLlaRY. If $f: D \rightarrow D^{\prime}$ is an n-dimensional $K$-quasiconformal mapping and if $A \subset D$ is contained in a countable union of $K$-quasiconformal $(n-1)$-balls, then $\mathrm{H}-\operatorname{dim} f[A] \leqslant \beta<n$, where $\beta$ depends only on $n$ and $K$.

Proof. Since $f$ is $K$-quasiconformal, $f[A]$ is contained in a countable union of $K^{2}$-quasiconformal $(n-1)$-balls, and the conclusion follows from Theorem 18 .

20. THEOREM. For each pair of integers $n \geqslant 2$ and $m \in[1, n-1]$ and each number $\beta \in[m, n)$, there exists a quasiconformal $m$-ball $S \subset R^{n}$ with $\mathrm{H}$-dim $S=\beta$.

Proof. Let $A=Q \cap T$, where $Q$ is the closed unit cube and $T$ is the $m$-plane

$$
T=\left\{x=\left(x_{1}, \ldots, x_{n}\right): x_{m+1}=\ldots=x_{n}=\frac{1}{2}\right\},
$$

and set $s=\left(4^{n}+1\right)^{-1}$. Then we can find $2^{n}$ disjoint oriented closed cubes $Q_{i}$ of side $s$ in int $Q$ with centres in $A$. Following the construction in $\$ 4$ for the corresponding Cantor set $C_{s}^{n}$, we see that for each $j$, the $2^{j n}$ disjoint closed cubes of side $s^{j}$ in $F_{j}$ all have their centres in $A$. Hence

$$
C_{s}{ }^{n}=\bigcap_{j=1}^{\infty} F_{j} \subset A .
$$

Now choose $t \in\left(0, \frac{1}{2}\right)$ and a Cantor set $C_{t}{ }^{n}$ so that H-dim $C_{t}{ }^{n}=\beta$, let $f: R^{n} \rightarrow R^{n}$ be the quasiconformal mapping given in the proof of Theorem 5 which maps $C_{s}{ }^{n}$ onto $C_{t}^{n}$, and set $S=f[A]$. Since $A$ is obviously a quasiconformal $m$-ball, so is $S$. Then (18) implies that

$$
A=\left(\bigcup_{j=1}^{\infty}\left(A \sim F_{j}\right)\right) \cup C_{s}{ }^{n},
$$

and hence that

$$
S=\left(\bigcup_{j=1}^{\infty} f\left[A \sim F_{j}\right]\right) \cup C_{t}^{n} .
$$


From the construction in the proof of Theorem 5, we see for each $j$ that $f(x)=f_{j}(x)$ in $R^{n} \sim F_{j}$ and hence that $f \mid\left(R^{n} \sim F_{j}\right)$ is piecewise linear. Thus

$$
\mathrm{H}-\operatorname{dim} f\left[A \sim F_{j}\right]=m \leqslant \beta
$$

for each $j$ and $\mathrm{H}-\operatorname{dim} S=\mathrm{H}-\operatorname{dim} C_{t}{ }^{n}=\beta$.

21. Remarks. Theorem 20 shows that the upper bound $\beta$ in Theorem 18 and Corollary 19 cannot be chosen so that it depends only on $n$.

We can also apply Theorem 20 to the theory of quasiregular mappings. Suppose that $f: D \rightarrow R^{n}$ is a quasiregular mapping, and let $B_{f}$ denote the branch set of $f$. Then $B_{f}$ and $f\left[B_{f}\right]$ are of $n$-dimensional measure zero [9;2.27 and 8.3]. Suppose next that $B_{f} \neq \varnothing$. Then from $[11 ; 3.4]$ it follows that $H_{n-2}\left(f\left[B_{f}\right]\right)>0$. Hence in this case

$$
n-2 \leqslant \mathrm{H}-\operatorname{dim} f\left[B_{f}\right] \leqslant n .
$$

On the other hand, by a result of Černavskii [2] (see also [15]), the topological dimension of $B_{f}$ is at most $n-2$, and the same is true for $f\left[B_{f}\right]$ by $[3 ; 2.2]$.

22. Corollary. For each integer $n \geqslant 3$ and each pair of numbers $\alpha, \beta \in[n-2, n)$, there exists a quasiregular mapping $f: R^{n} \rightarrow R^{n}$ such that

$$
\mathrm{H}-\operatorname{dim} B_{f}=\alpha, \mathrm{H}-\operatorname{dim} f\left[B_{f}\right]=\beta .
$$

Proof. Set $m=n-2$ and let $T$ be the (n-2)-plane in (17). Since (19) holds with $A$ replaced by $T$, the proof of Theorem 20 shows that we can construct quasiconformal mappings $g_{1}: R^{n} \rightarrow R^{n}$ and $g_{2}: R^{n} \rightarrow R^{n}$ such that

$$
\mathrm{H}-\operatorname{dim} g_{1}[T]=\alpha, \mathrm{H}-\operatorname{dim} g_{2}[T]=\beta \text {. }
$$

Next define as in $[11 ; 3.19]$ a quasiregular winding mapping $h: R^{n} \rightarrow R^{n}$ with $B_{h}=h\left[B_{h}\right]=T$, and set $f=g_{2} \circ h \circ g_{1}{ }^{-1}$. Then $f: R^{n} \rightarrow R^{n}$ is quasiregular and $B_{f}=g_{1}[T], f\left[B_{f}\right]=g_{2}[T]$.

23. Final remarks. The argument in the proof of Theorem 5 can be used to show that the lower bound in Theorem 8 is asymptotically sharp for sets of small Hausdorff dimension. More precisely, given $K \in(1, \infty)$, one can construct for each $\alpha \in(0, n)$ a $K$-quasiconformal mapping $f_{\alpha}: R^{n} \rightarrow R^{n}$ and a compact set $A_{\alpha} \subset R^{n}$ with

such that

$$
\text { H-dim } A_{\alpha}=\alpha
$$

$$
\lim _{\alpha \rightarrow 0} \frac{\mathrm{H}-\operatorname{dim} f_{\alpha}\left[A_{\alpha}\right]}{\alpha}=K^{1 /(1-n)} .
$$

This argument also can be used to show that for each $K \in(1, \infty)$ there exists a $K$-quasiconformal mapping $f: R^{n} \rightarrow R^{n}$ and a compact set $A \subset R^{n}$ with H-dim $A=n$ such that $f$ is differentiable with a vanishing Jacobian at each point of $A$.

\section{References}

1. A. F. Beardon, "On the Hausdorff dimension of general Cantor sets ", Proc. Cambridge Philos. Soc., 61 (1965), 679-694.

2. A. V. Cernavskii, "Finite to one open mappings of manifolds", Mat. Sbornik, 65 (1964), 357-369. (Russian.) 
3. P. T. Church and E. Hemmingsen, "Light open maps on n-manifolds", Duke Math. J., 27 (1960), 527-536.

4. F. W. Gehring, "Symmetrization of rings in space ", Trans. Amer. Math. Soc., 101 (1961), 499-519.

5. __ , "Rings and quasiconformal mappings in space ", Trans. Amer. Math. Soc., 103 (1962), 353-393.

6. W. Hurewicz and H. Wallman, Dimension theory (Princeton Univ. Piess, 1941).

7. O. Lehto and K. I. Virtanen, Quasikonforme Abbildungen (Springer Verlag, 1965).

8. J. M. Marstrand, "The dimension of Cartesian product sets", Proc. Cambridge Philos. Soc., 50 (1954), 198-202.

9. O. Martio, S. Rickman and J. Väisälä, "Definitions for quasiregular mappings ", Ann. Acad. Sci. Fenn. A I, 448 (1969), 1-40.

10. — - - and $\longrightarrow$, Distortion and singularities of quasiregular mappings", Ann. Acad. Sci. Fenn. A I, 465 (1970), 1-13.

11. - _ Ann. Acad. Sci. Fenn. A I, 488 (1971), 1-31.

12. P. A. P. Moran, "Additive functions of intervals and Hausdorff measure", Proc. Cambridge Philos. Soc., 42 (1946), 15-23.

13. A. Mori, "On quasi-conformality and pseudo-analyticity", Trans. Amer. Math. Soc., 84 (1957), 56-77.

14. G. D. Mostow, "Quasi-conformal mappings in $n$-space and the rigidity of hyperbolic space forms", Inst. Hautes Etudes Sci. Publ. Math., 34 (1968), 53-104.

15. J. Väisälä, “ Discrete open mappings on manifolds ”, Ann. Acad. Sci. Fenn. A I, 392 (1966), 1-10.

16. __ " "Lectures on $n$-dimensional quasiconformal mappings", Lecture notes in mathematics 229 (Springer Verlag, 1971).

University of Michigan

University of Minnesota

University of Helsinki 